\title{
The Meyer property of cut-and-project sets
}

\author{
Lubomíra Balková, Zuzana Masákováł and Edita Pelantová \\ Department of Mathematics, Faculty of Nuclear Sciences and Physical Engineering, \\ Czech Technical University, Trojanova 13, 12000 Praha 2, Czech Republic
}

\begin{abstract}
We consider cut-and-project sets $\Sigma(\Omega)$ with compact acceptance window $\Omega \subset \mathbb{R}^{d}$. It is known that $\Sigma(\Omega)$ satisfies the Meyer property, i.e. is a Delone set and there exists a finite set $F$ such that $\Sigma(\Omega)-\Sigma(\Omega) \subset \Sigma(\Omega)+F$. The investigation of the set $F$ can be transformed to the problem of covering of the the difference set $\Omega-\Omega$ by open copies $\Omega^{\circ}$. The cardinality $f(\Omega)$ of the minimal covering is called the Meyer number of $\Omega$. We study topological properties of the function $f$ and show that it is bounded on the space of convex compact sets $\Omega \subset \mathbb{R}^{d}$. We give estimates on the universal upper bound of the Meyer number of $\Omega \subset \mathbb{R}^{2}$. We further show that $f$ is not bounded if we relax the condition of convexity.
\end{abstract}

Submitted to: J. Phys. A: Math. Gen.

$\ddagger$ Corresponding author (masakova@km1.fjfi.cvut.cz) 


\section{Introduction}

Since the discovery of quasicrystals, their modeling has been the driving task for theoretical studies of the subject. In general, a mathematical object representing atomic positions in a material is a point set $\Lambda \subset \mathbb{R}^{n}$ constrained by two simple physically reasonable properties - discreteness and homogenity. The common basis for all the constructions is that $\Lambda$ should satisfy the Delone property.

Definition 1.1. A set $\Lambda \subset \mathbb{R}^{n}$ is Delone, if there exist $r_{1}, r_{2}>0$ such that

(i) $\Lambda$ is uniformly discrete: $\|x-y\| \geq r_{1}$ for any $x, y \in \Lambda, x \neq y$.

(ii) $\Lambda$ is relatively dense: $B\left(x, r_{2}\right) \cap \Lambda \neq \emptyset$ for any $x \in \mathbb{R}^{n}$, where $B\left(x, r_{2}\right)$ is the $n$-dimensional ball of radius $r_{2}$ centered at $x$.

A simple example of a Delone set in $\mathbb{R}^{n}$ is a lattice, i.e. $\Lambda=\left\{\sum_{i=1}^{n} a_{i} x_{i} \mid a_{i} \in \mathbb{Z}\right\}$ for any basis $x_{1}, \ldots, x_{n}$ of $\mathbb{R}^{n}$. Lattices are characterized

$$
\Lambda-\Lambda \subset \Lambda
$$

which correspond to the fact that they have translational symmetries and therefore serve for models of periodic crystals.

For models of quasicrystalline structures Meyer [6] proposed a concept that generalizes lattices. He calls a 'quasicrystal' a Delone set $\Lambda$ which satisfies the property of 'almost-lattices'

$$
\Lambda-\Lambda \subset \Lambda+F
$$

for some finite set F. Moody [7] calls such sets 'Meyer sets' and shows that they are precisely Delone harmonious sets. Lagarias in [4] provides yet another equivalent definition of a Meyer set, i.e. a Delone set $\Lambda$ such that $\Lambda-\Lambda$ is also Delone. One of the nice properties of Meyer sets is that they have finite local complexity, which means that for every $\varrho>0$ there are, up to translation, finitely many 'patches' of the form $(\Lambda \cap B(x, \varrho))-x$, for $x \in \Lambda$. Every such patch is embedded in

$$
(\Lambda-\Lambda) \cap B(0, \varrho) \quad \subset \quad(\Lambda+F) \cap B(0, \varrho)
$$

Therefore the structural complexity of the point set $\Lambda$ depends on the cardinality of $F$.

An important topic in quasicrystal theory is diffraction described by the Fourier transform $\hat{\gamma}$ of the autocorrelation $\gamma$ of the measure $\nu$ that represents atomic density. What is required from mathematical models of quasicrystalline structures is that $\hat{\gamma}$ has a discrete part, which corresponds to bright spots on the diffraction diagram that physicists call 'Bragg peaks'.

The relation of Meyer sets to diffraction is not at all obvious. Therefore the focus has been made on the so-called cut-and-project sets which form a rich class of Meyer sets that might be considered quasicrystalline in the sense of having some discrete component in their diffraction spectrum. Their importance in modeling quasicrystals has been pointed out by Kramer [3]. Cut-and-project sets arise as a projection of chosen points 
of a higher-dimensional lattice on a lower-dimensional 'physical space'. The choice of lattice point is controlled by an 'acceptance window' in the non-physical 'inner space'. An important result of Hof [2] is that any regular cut-and-project set diffracts, i.e. has a pure point spectrum supported on a finitely generated $\mathbb{Z}$-module.

The definition of cut-and-project sets which we provide below is not the most general one (for that see $[6,7]$ ). In our considerations both physical and inner spaces are Euclidean. Patera in [8] shows, how to choose the lattice and the projection, in order to obtain quasicrystal models with 5 -fold symmetries, which have been observed in nature [9]. For general introduction to quasicrystals and related problems we refer to $[10]$.

Definition 1.2. Let $V_{1}$ and $V_{2}$ be non-trivial subspaces of $\mathbb{R}^{n}$ such that $V_{1} \oplus V_{2}=\mathbb{R}^{n}$, the restriction of $\pi_{1}$ on the lattice $\mathbb{Z}^{n}$ is one-to-one, and $\pi_{2}\left(\mathbb{Z}^{n}\right)$ is dense in $V_{2}$, where $\pi_{1}$, $\pi_{2}$ are the projections on $V_{1}$ along $V_{2}$, and $V_{2}$ along $V_{1}$, respectively. Let $\Omega$ be a compact set with non-empty interior $\Omega^{\circ}$. The set

$$
\Sigma(\Omega):=\left\{\pi_{1}(x) \mid x \in \mathbb{Z}^{n}, \pi_{2}(x) \in \Omega\right\}
$$

is called a cut-and-project set with acceptance window $\Omega$.

The conditions we impose on the acceptance window influence the properties of the cut-and-project set $\Sigma(\Omega)$. For example for diffractivity one needs that $\Omega$ has a boundary of Lebesgue measure zero (such cut-and-project set is called regular). Meyer showed that all cut-and-project sets are Delone and satisfy the condition (1) of almostlattices, (i.e. are Meyer sets). On the other hand, he showed that a Delone set $\Lambda$ is a Meyer set if and only if there exists a cut-and-project set $\Sigma(\Omega)$ and a finite set $F$ such that $\Lambda \subset \Sigma(\Omega)+F$. Since all cut-and-project sets are Meyer sets, they have finite local complexity. Moreover, if the boundary $\partial \Omega$ of the acceptance window $\Omega$ satisfies $\partial \Omega \cap \pi_{2}\left(\mathbb{Z}^{n}\right)=\emptyset$, the cut-and-project set is repetitive, which means that every patch that appears in $\Sigma(\Omega)$, appears infinitely often.

In our paper we focus on the Meyer property of cut-and-project sets, in particular, the cardinality of the finite set $F$ satisfying $\Sigma(\Omega)-\Sigma(\Omega) \subset \Sigma(\Omega)+F$ in dependence on the choice of acceptance window $\Omega$. In Section 2 we show that the problem can be transformed to studying the property $\Omega-\Omega \subset \Omega+G$ for a finite set $G$. We denote the cardinality of the minimal set $G$ by $f(\Omega)$ and call it the Meyer number of $\Omega$. We show in Section 3 that on the space of all convex acceptance windows $\Omega$ the Meyer number is bounded. Moreover, the function $f$ on this space equipped with the Hausdorff metric is upper semi-continuous, see Section 4. In Section 5 we show that relaxing the condition of convexity, boundedness of $f$ is no longer true. In Section 6 we provide estimates on the universal upper bound on the Meyer number in dimension two. For special types of acceptance windows we determine $f(\Omega)$, see Section 7 . 


\section{Meyer property of cut-and-project sets}

As we have recalled, every cut-and-project set satisfies the Meyer property, i.e. is Delone and

$$
\Sigma(\Omega)-\Sigma(\Omega) \subset \Sigma(\Omega)+F
$$

for some finite set $F$. Obviously, $F \subset \pi_{1}\left(\mathbb{Z}^{n}\right)$ and for the finite set $G:=\pi_{2} \pi_{1}^{-1}(F)$ we have

$$
\Omega-\Omega \subset \Omega+G \text {. }
$$

The converse is however not that simple. Having $G \subset V_{2}$ which satisfies (3), it is not always possible to find $F$ of the same cardinality, so that (2) holds, which comes from the fact that $G$ may not be a subset of $\pi_{2}\left(\mathbb{Z}^{n}\right)$. However, this inconvenience can be avoided if we study coverings of the difference set $\Omega-\Omega$ by copies of the interior $\Omega^{\circ}$.

$$
\overline{\Omega-\Omega} \subset \Omega^{\circ}+G .
$$

Having such $G$ and due to the fact that $\pi_{2}\left(\mathbb{Z}^{n}\right)$ is dense in $V_{2}$, we can clearly find a set $\tilde{G} \subset \pi_{2}\left(\mathbb{Z}^{n}\right)$ of the same cardinality as $G$ and satisfying (3). Therefore we may set $F=\pi_{1} \pi_{2}^{-1}(\tilde{G})$ to obtain $(2)$ with $|F|=|G|$.

For compact sets $\Omega \subset \mathbb{R}^{d}$ with non-empty interior we are interested in the cardinality of the minimal finite set $G$ satisfying (4). This cardinality is denoted by $f(\Omega)$ and called the Meyer number of $\Omega$. Formally,

$$
f(\Omega):=\min \left\{k \in \mathbb{N} \mid \exists G \subset \mathbb{R}^{d}, \text { satisfying } \overline{\Omega-\Omega} \subset \Omega^{\circ}+G \text { and }|G|=k\right\} .
$$

The main result of the paper is stated as follows.

Theorem 2.1. For every dimension $d \in \mathbb{N}$, there exist a constant $K_{d} \in \mathbb{N}$, such that for all convex compact sets $\Omega \subset \mathbb{R}^{d}$ we have $f(\Omega) \leq K_{d}$.

\section{Proof of boundedness of the function $f$}

In this section we provide the proof of Theorem 2.1, which says that the function $f$ is bounded on the space of convex compact sets with non-empty interior in $\mathbb{R}^{d}$. We first need to show an important property of the function $f$, namely that $f$ is invariant under affine transformations of $\Omega$. More precisely, we have the following proposition.

Proposition 3.1. Let $A: \mathbb{R}^{d} \rightarrow \mathbb{R}^{d}$ be a bijective affine map. Then $f(A \Omega)=f(\Omega)$ for every convex compact set $\Omega \subset \mathbb{R}^{d}$ with non-empty interior.

Proof. Clearly, the value of $f$ is invariant under translation of $\Omega$, since $(\Omega+x)-(\Omega+x)=$ $\Omega-\Omega$ for all $x \in \mathbb{R}^{d}$. Thus we can consider without loss of generality $A$ to be a nonsingular linear map. If $\Omega$ satisfies $\overline{\Omega-\Omega} \subset\left(a_{1}+\Omega^{\circ}\right) \cup \cdots \cup\left(a_{k}+\Omega^{\circ}\right)$, then

$$
\begin{aligned}
\overline{A \Omega-A \Omega} & =\overline{\overline{(\Omega-\Omega)}} \subset\left(A a_{1}+A \Omega^{\circ}\right) \cup \cdots \cup\left(A a_{k}+A \Omega^{\circ}\right)= \\
& =\left(A a_{1}+(A \Omega)^{\circ}\right) \cup \cdots \cup\left(A a_{k}+(A \Omega)^{\circ}\right),
\end{aligned}
$$


where we have used that for a non-singular map $A$ it holds that $A \Omega^{\circ}=(A \Omega)^{\circ}$ and $A \bar{\Omega}=\overline{A \Omega}$. Therefore $f(A \Omega) \leq f(\Omega)$. Since this was valid for arbitrary $A$ and arbitrary $\Omega$, one has also $f(\Omega)=f\left(A^{-1} A \Omega\right) \leq f(A \Omega)$, which completes the proof.

Crucial for the proof of Theorem 2.1 is the following assertion taken from [5].

Theorem 3.2 (John). For every convex compact set $\Omega$ with non-empty interior in $\mathbb{R}^{d}$ there exists a closed ellipsoid $E$ such that $E+z \subset \Omega \subset d E+z$, where $z \in \mathbb{R}^{d}$.

Corollary 3.3. Let $\Omega$ be a convex compact set with non-empty interior in $\mathbb{R}^{d}$. Then $f(\Omega)$ is smaller or equal to the number of copies $B(0,1)$ which are needed for covering $\overline{B(0,2 d)}$.

Proof. Using Theorem 3.2 there exists a closed ellipsoid $E \subset \mathbb{R}^{d}$ such that $E+z \subset$ $\Omega \subset d E+z$. We find a non-singular affine map $A$ such that $A(E+z)=\overline{B(0,1)}$ and $A(d E+z)=\overline{B(0, d)}$. Then $B(0,1) \subset(A \Omega)^{\circ} \subset B(0, d)$. If $n$ is the number of copies of $B(0,1)$ needed to cover $B(0,2 d)$, i.e.

$$
\overline{B(0,2 d)} \subset\left(x_{1}+B(0,1)\right) \cup \cdots \cup\left(x_{n}+B(0,1)\right)
$$

for some $x_{1}, \ldots, x_{n} \in \mathbb{R}^{d}$, then

$$
\begin{aligned}
A \Omega-A \Omega & \subset \overline{B(0, d)-B(0, d)}=\overline{B(0,2 d)} \subset\left(x_{1}+B(0,1)\right) \cup \cdots \cup\left(x_{n}+B(0,1)\right) \subset \\
& \subset\left(x_{1}+(A \Omega)^{\circ}\right) \cup \cdots \cup\left(x_{n}+(A \Omega)^{\circ}\right) .
\end{aligned}
$$

Therefore using Proposition 3.1 we obtain $f(\Omega)=f(A \Omega) \leq n$ what was to show.

Proof of Theorem 2.1. Using Corollary 3.3 it suffices to show that for every dimension $d$ only a finite number of copies of $B(0,1) \subset \mathbb{R}^{d}$ are needed for covering $\overline{B(0,2 d)}$. This is clear since as a consequence of compactness of $\overline{B(0,2 d)}$ we can find a finite subcovering of the covering

$$
\overline{B(0,2 d)} \subset \bigcup_{x \in \overline{B(0,2 d)}} B(x, 1)
$$

Thus $f$ is bounded on the space of convex compact sets in $\mathbb{R}^{d}$ with non-empty interior.

\section{Semi-continuity of $f$}

In this section we show that the function $f$ is upper-semi-continuous. We explain, how this fact can serve for an alternative proof of boundedness of $f$. It would be an interesting problem to study the division of the space of all convex compact sets $\Omega \subset \mathbb{R}^{d}$ into classes according to the value $f(\Omega)$. Semi-continuity of $f$ may well serve this purpose.

Let us first state several facts from topology of spaces of convex sets that will be needed. Consider $\mathbb{R}^{d}$ with Euclidean norm $\|\cdot\|$. One defines the distance of the set $\Omega$ from the point $x$ as

$$
\rho(x, \Omega):=\inf \{\|x-y\| \mid y \in \Omega\}
$$


An $\varepsilon$-neighbourhood of the set $\Omega(\varepsilon>0)$ is the set

$$
\Omega_{\varepsilon}:=\left\{x \in \mathbb{R}^{2} \mid \rho(x, \Omega)<\varepsilon\right\} .
$$

Clearly, $\Omega_{\varepsilon}$ is an open set in $\mathbb{R}^{d}$. Distance of two sets in $\mathbb{R}^{2}$ is defined as

$$
\operatorname{dist}(\Omega, \tilde{\Omega}):=\inf \left\{\varepsilon>0 \mid \Omega \subset \tilde{\Omega}_{\varepsilon} \text { and } \tilde{\Omega} \subset \Omega_{\varepsilon}\right\} .
$$

Remark 4.1. Recall that $\operatorname{dist}(\Omega, \tilde{\Omega})$ is the so-called Hausdorff metric on the space of all compact subsets of $\mathbb{R}^{d}$. For more on topologies of compact sets see [1].

Let us define $(-\varepsilon)$-neighbourhood of a bounded convex set $\Omega$, for $\varepsilon>0$,

$$
\Omega_{-\varepsilon}:=\{x \in \Omega \mid \rho(x, \partial \Omega)>\varepsilon\},
$$

where $\partial \Omega$ denotes the boundary of $\Omega$. It is obvious from the definition that $\Omega_{-\varepsilon}$ is an open set.

Lemma 4.2. Let $\Omega$ and $\tilde{\Omega}$ be bounded closed and convex subsets of $\mathbb{R}^{2}$, such that $\operatorname{dist}(\Omega, \tilde{\Omega})<\delta$. Then

$$
\Omega_{-\delta} \subset \tilde{\Omega} \subset \Omega_{\delta}
$$

Proof. The inclusion $\tilde{\Omega} \subset \Omega_{\delta}$ is valid for any bounded sets $\Omega$ and $\tilde{\Omega}$ directly from the definition of $\delta$-neighbourhood of a set. The other inclusion, $\Omega_{-\delta} \subset \tilde{\Omega}$ can be shown par absurdum. Assume that there exists $x \in \Omega_{-\delta}$ such that $x \notin \tilde{\Omega}$. The convexity of $\Omega$ and the definition of $\Omega_{-\delta}$ imply that $B(x, \delta) \subset \Omega$. Since $\tilde{\Omega}$ is closed and convex, and $x \notin \tilde{\Omega}$, there exists a hyperplane $H$, such that $\tilde{\Omega}$ is all contained in one of the half-spaces bounded by $H$, and that $x$ belongs to the other half-space. Therefore at least one entire half of the ball $B(x, \delta)$ does not belong to $\tilde{\Omega}$. This is a contradiction with $\Omega \subset \tilde{\Omega}_{\delta}$.

Theorem 4.3. On the family of all compact convex sets with non-empty interior the function $f$ is upper semi-continuous, i.e for any $\Omega$ there exists $\delta>0$, such that for any $\tilde{\Omega}$ with the property $\operatorname{dist}(\Omega, \tilde{\Omega})<\delta$ one has

$$
f(\tilde{\Omega}) \leq f(\Omega) .
$$

Proof. Let $f(\Omega)=k \in \mathbb{N}$ and $\overline{\Omega-\Omega} \subset P:=\left(a_{1}+\Omega^{\circ}\right) \cup \cdots \cup\left(a_{k}+\Omega^{\circ}\right)$. Put

$$
\varepsilon:=\inf \left\{\rho\left(x, \mathbb{R}^{2} \backslash P\right) \mid x \in \overline{\Omega-\Omega}\right\} .
$$

Necessarily $\varepsilon>0$ and one has $\overline{(\Omega-\Omega)_{\varepsilon / 2}} \subset P$. For any $x \in \overline{(\Omega-\Omega)_{\varepsilon / 2}}$ we find those of $a_{i}+\Omega^{\circ}$ which contain $x$, say

$$
x \in a_{i_{s}}+\Omega^{\circ}, \quad \text { for } s=1,2, \ldots, r .
$$

Let us define

$$
v(x):=\max \left\{\rho\left(x, \partial\left(a_{i_{s}}+\Omega^{\circ}\right)\right) \mid s=1,2, \ldots, r\right\} .
$$


Clearly, $v(x)>0$. Moreover, $v(x)$ is a continuous function on the compact $\overline{(\Omega-\Omega)_{\varepsilon / 2}}$. Therefore it has there its minimum, say $\nu$,

$$
\forall x \in \overline{(\Omega-\Omega)_{\varepsilon / 2}}: \quad v(x) \geq \nu>0 .
$$

This implies

$$
\overline{(\Omega-\Omega)_{\varepsilon / 2}} \subset\left(a_{1}+\Omega_{-\nu / 2}\right) \cup \cdots \cup\left(a_{k}+\Omega_{-\nu / 2}\right) .
$$

In order to complete the proof of Proposition 4.3, put $\delta=\min \left\{\frac{\varepsilon}{4}, \frac{\nu}{2}\right\}$. Let now $\operatorname{dist}(\Omega, \tilde{\Omega})<\delta$. Then

$$
\begin{aligned}
\overline{\tilde{\Omega}-\tilde{\Omega}} & \subset \overline{\Omega_{\delta}-\Omega_{\delta}} \subset \overline{(\Omega-\Omega)_{2 \delta}} \subset \overline{(\Omega-\Omega)_{\varepsilon / 2}} \subset \\
& \subset\left(a_{1}+\Omega_{-\nu / 2}^{\circ}\right) \cup \cdots \cup\left(a_{k}+\Omega_{-\nu / 2}^{\circ}\right) \subset \\
& \subset\left(a_{1}+\Omega_{-\delta}^{\circ}\right) \cup \cdots \cup\left(a_{k}+\Omega_{-\delta}^{\circ}\right) \subset \\
& \subset\left(a_{1}+\tilde{\Omega}^{\circ}\right) \cup \cdots \cup\left(a_{k}+\tilde{\Omega}^{\circ}\right) .
\end{aligned}
$$

This means that $f(\tilde{\Omega}) \leq k=f(\Omega)$. Note that in the sequence of inclusions we have used Lemma 4.2 and two obvious facts, namely that $\Omega_{-\beta} \subset \Omega_{-\alpha}$ and $\Omega_{\alpha} \subset \Omega_{\beta}$ for $0<\alpha<\beta$, and that $\Omega_{\delta}-\Omega_{\delta} \subset(\Omega-\Omega)_{2 \delta}$ for $\delta>0$.

Theorem 4.3 can be used for an alternative proof of Theorem 2.1 as follows. For an arbitrary convex compact set $\Omega \subset \mathbb{R}^{d}$ with non-empty interior according to Theorem 3.2 there exists a closed ellipsoid $E$ such that $E+z \subset \Omega \subset d E+z$ for some $z \in \mathbb{R}^{d}$. Using Proposition 3.1 we can find a convex compact set $\tilde{\Omega}$ satisfying

$$
\overline{B(0,1)} \subset \tilde{\Omega} \subset \overline{B(0, d)}
$$

with the same Meyer number as $\Omega, f(\tilde{\Omega})=f(\Omega)$. It is not difficult to show that the space of convex compact $\tilde{\Omega} \subset \mathbb{R}^{d}$ satisfying (6) is a compact space with the Hausdorff metric. Since the function $f$ is upper semi-continuous on this compact space, $f$ is bounded from above on it, and therefore it is also bounded on the space of arbitrary convex $\Omega$ with non-empty interior.

\section{Unboundedness of $f$ on the space of general compact sets}

Sofar we have treated only convex compact sets in $\mathbb{R}^{d}$. We stand in front of a natural question. Is the function $f$ bounded even if we relax the condition of convexity? The answer is negative.

Proposition 5.1. There exists a sequence $\left(\Omega_{n}\right)_{n \in \mathbb{N}}$ of compact sets in $R^{d}$ with nonempty interior, such that $\lim _{n \rightarrow \infty} f\left(\Omega_{n}\right)=+\infty$.

Proof. We construct the counterexample of boundedness of the function $f$ in dimension 2. Generalization to higher dimensions is straightforward. Let $\Omega_{n}$ be compact sets with 
non-empty interior containing the line segments $\{(t, 0) \mid t \in[-1,1]\},\{(0, t) \mid t \in[-1,1]\}$ for all $n \in \mathbb{N}$, and such that $\lim _{n \rightarrow \infty} \operatorname{vol}\left(\Omega_{n}\right)=0$.

Then obviously $\Omega_{n}-\Omega_{n}$ contains the square of side length 1 centered at the origin, $\left\{\left(t_{1}, t_{2}\right) \mid t_{1}, t_{2} \in[-1,1]\right\}$. For the volume of $\Omega_{n}-\Omega_{n}$ we thus have $\operatorname{vol}\left(\Omega_{n}-\Omega_{n}\right) \geq 4$. Therefore

$$
f\left(\Omega_{n}\right) \geq \frac{\operatorname{vol}\left(\Omega_{n}-\Omega_{n}\right)}{\operatorname{vol}\left(\Omega_{n}\right)} \geq \frac{4}{\operatorname{vol}\left(\Omega_{n}\right)},
$$

which implies $\lim _{n \rightarrow \infty} f\left(\Omega_{n}\right)=+\infty$, what we wanted to show.

Let us mention that we can construct the counterexample even on the sets which are the nearest generalization of convex sets, namely star-shaped sets. (We say that $\Omega \subset \mathbb{R}^{d}$ is star-shaped, if there exists an $x \in \mathbb{R}^{d}$ such that $\lambda x+(1-\lambda) y \in \Omega$ for every $y \in \Omega$ and all $\lambda \in(0,1)$.) An example of a sequence of star-shaped sets $\Omega_{n}$ satisfying $\lim _{n \rightarrow \infty} f\left(\Omega_{n}\right)=+\infty$ and the corresponding difference sets $\Omega_{n}-\Omega_{n}$ can be found in Figure 1.
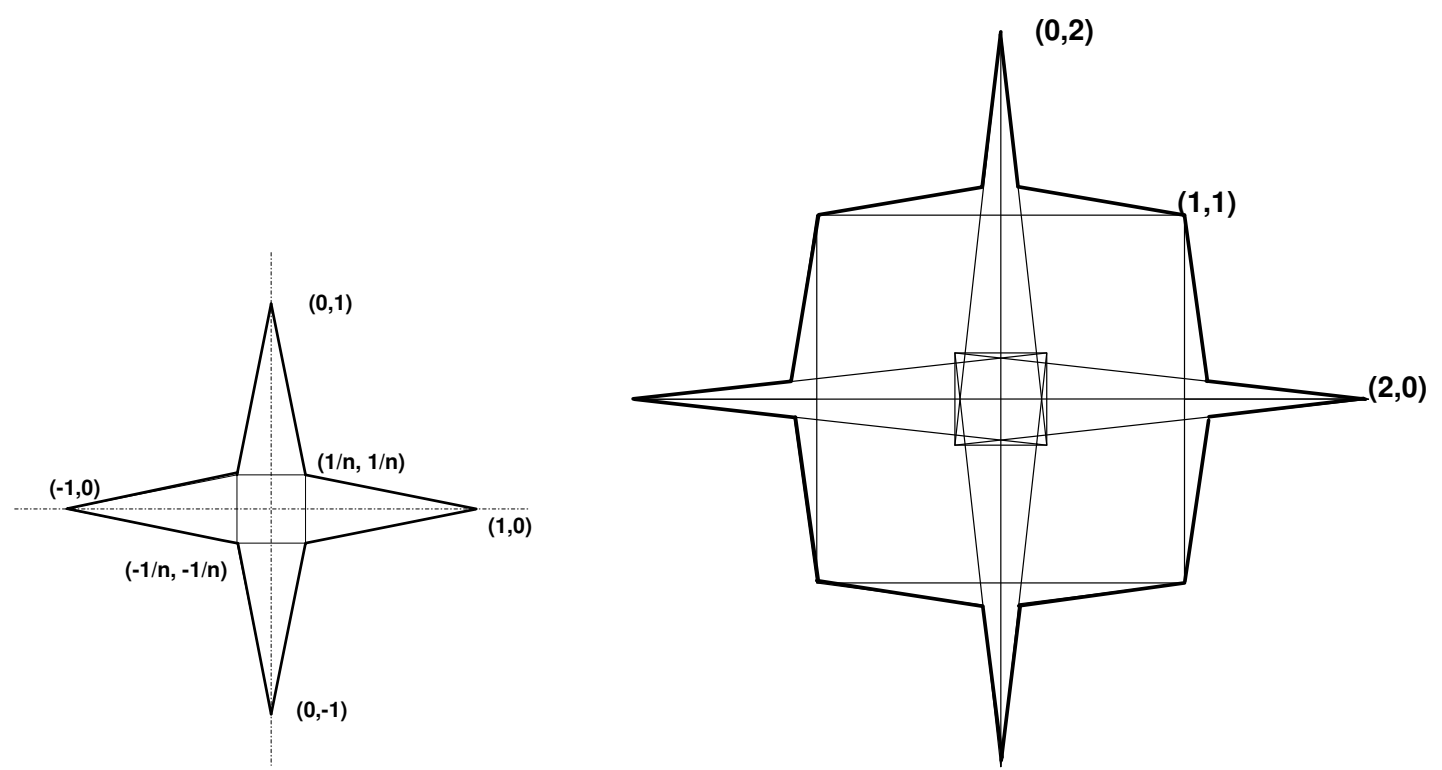

Figure 1. Illustration of a sequence of star-shaped sets $\Omega_{n}, n \in \mathbb{N}$, satisfying $\lim _{n \rightarrow \infty} f\left(\Omega_{n}\right)=+\infty$. The left hand part of the figure shows $\Omega_{n}$, on the right hand side there is $\Omega_{n}-\Omega_{n}$.

Note also that we have omitted the proof of unboundedness of $f$ in dimension one. There every star-shaped set is convex, the counterexample in $\mathbb{R}$ must therefore be built on non-connected sets. The construction of such a sequence of sets is simple but technical.

\section{Universal bound on the Meyer number for convex sets in the plane}

In this section we provide an estimate on the value of the function $f$ for two-dimensional convex compact sets $\Omega$. Corollary 3.3 gives us a method to find the universal bound $K_{d}$ 
on the Meyer number of convex sets in $\mathbb{R}^{d}$. For the case $d=2$ we have to determine the number of copies of the open unit ball $B(0,1)$ needed to cover the closed ball $\overline{B(0,4)}$. The result is stated in the following proposition.

Proposition 6.1. Let $\Omega$ be a convex compact set in $\mathbb{R}^{2}$. Then $f(\Omega) \leq K_{2} \leq 26$.

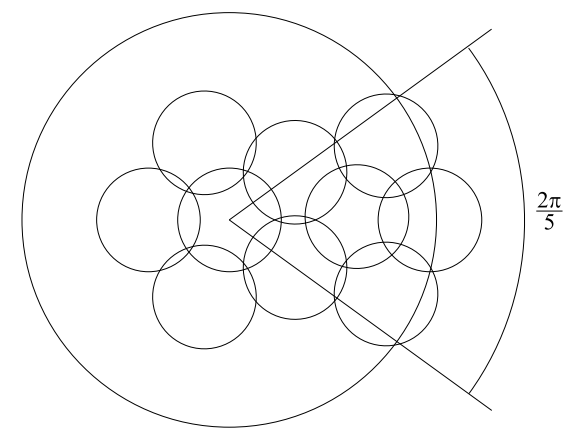

Figure 2. Illustration of proof of Proposition 6.1. Six copies of the unit ball are used to cover the central part of $\overline{B(0,4)}$. Four more copies are needed to cover the section of angle $2 \pi / 5$.

Proof. Figure 2 shows that it is possible to cover $\overline{B(0,4)}$ by 26 translated copies of the ball $B(0,1)$. Hence, $f(\Omega) \leq 26$ for every convex compact set $\Omega$ with non-empty interior in $\mathbb{R}^{2}$.

It is conceivable that the above estimate on the uniform upper bound on $f$ on the space of convex compact sets is too rough. We conjecture that the maximum of the function $f$ is reached on a triangle.

Remark 6.2. Any triangle $\Omega$ can be transformed to an equilateral one by an affine map. Therefore it suffices to determine the Meyer number of an equilateral triangle. If $\Omega$ is such a triangle with side-length 1 , then $\Omega-\Omega$ is a regular hexagon of radius 1 . Figure 3 shows that thirteen open copies of the triangle $\Omega$ are sufficient to cover the closed hexagon $\Omega-\Omega$, i.e. $f(\Omega) \leq 13$.

Most likely, 12 copies of a triangle are not sufficient to cover the hexagon and thus $f(\Omega)=13$. According to our knowledge, among all two-dimensional convex sets, the triangle has the largest Meyer number. On the other hand it is likely that $f(\Omega)$ is smallest for $\Omega$ being an ellipse.

Remark 6.3. For every closed ellipse $E$ in $\mathbb{R}^{2}$ there exists an affine mapping such that $A(E)=\overline{B(0,1)}$. Using Proposition 3.1 we have $f(E)=f(A(E))=f(\overline{B(0,1)})$. Figure 6 illustrates determining of the value of $f(\overline{B(0,1)})=f(E)=8$.

All together, we conjecture that $8 \leq f(\Omega) \leq 13$ for every convex compact set $\Omega \subset \mathbb{R}^{2}$

Naturally, we can suppose that imposing more conditions on the convex compact set $\Omega$ will improve the bound on the value of $f$. Let us study the case of centrally symmetric $\Omega$. For this purpose we cite another result of John [5]: 


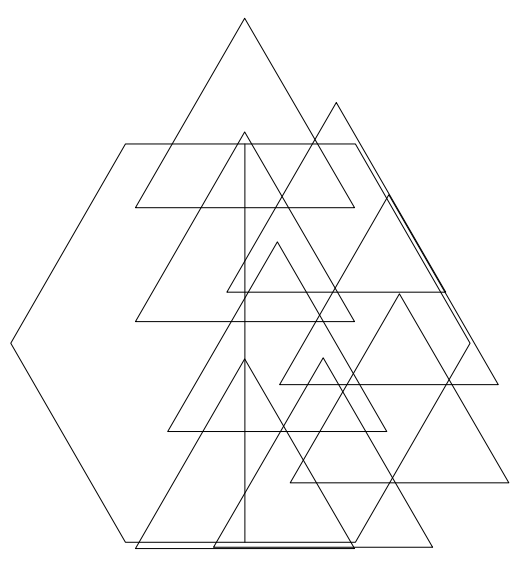

Figure 3. Let $\Omega$ be a triangle. Then $f(\Omega) \leq 13$.
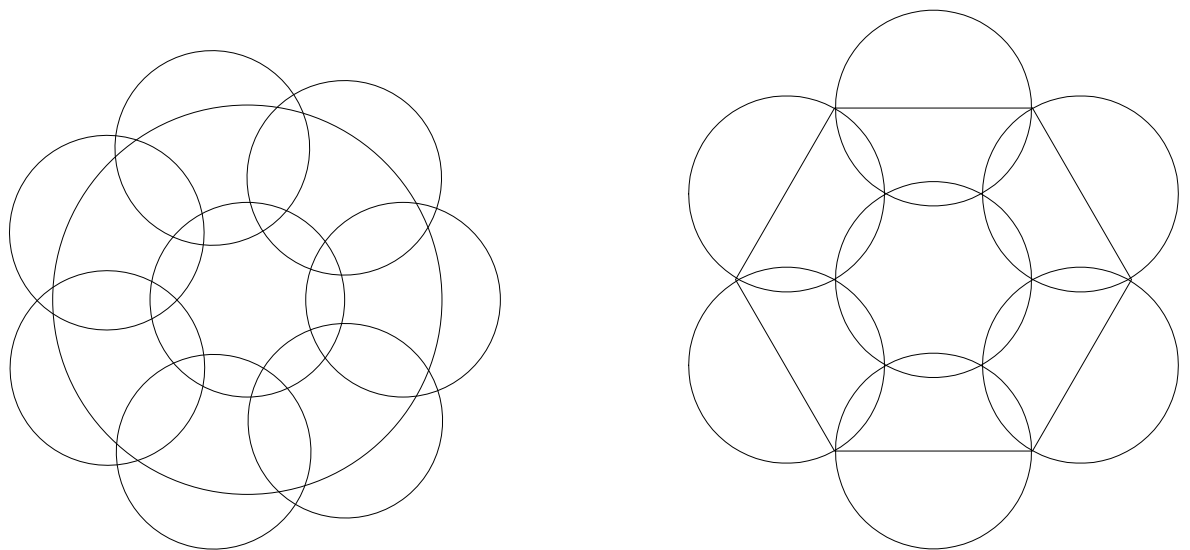

Figure 4. The left hand part of Figure 6 shows that eight copies of a unit ball are sufficient to cover $\overline{B(0,2)}$. The right hand part of the figure illustrates that seven are not sufficient, since for covering the boundary of $\overline{B(0,2)}$ one needs six closed and not only open unit balls. This means that if $\Omega$ is an ellipse, then $f(\Omega)=8$.

Theorem 6.4 (John). For every compact centrally symmetric set $\Omega$ with non-empty interior in $\mathbb{R}^{d}$ there exists a centrally symmetric ellipsoid $E$ such that $E \subset \Omega \subset \sqrt{d} E$.

In dimension $d=2$ this theorem has the following corollary. Its proof is analogous to the proof of Corollary 3.3.

Corollary 6.5. Let $\Omega$ be a convex compact centrally symmetric set with non-empty interior in $\mathbb{R}^{2}$. Then $f(\Omega)$ is smaller or equal to the number of copies $B(0,1)$ which are needed for covering $\overline{B(0,2 \sqrt{2})}$.

As a consequence, we can determine the upper bound on the value $f(\Omega)$ for all convex compact centrally symmetric sets $\Omega$ with non-empty interior.

Proposition 6.6. Let $\Omega$ be a centrally symmetric convex compact set in $\mathbb{R}^{2}$ with nonempty interior. Then $f(\Omega) \leq 16$. 


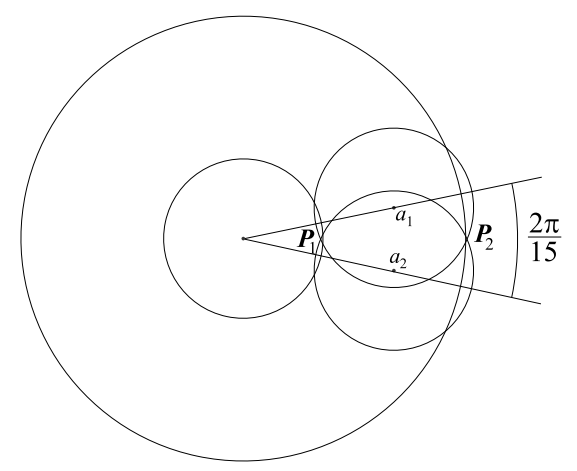

Figure 5. Illustration of proof of Proposition 6.6. For covering the boundary of $\overline{B(0,2 \sqrt{2})} 15$ translated copies of $B(0,1)$ are sufficient.

Proof. As a consequence of Corollary 6.5 it suffices to show that sixteen copies of the open unit ball are sufficient for covering $\overline{B(0,2 \sqrt{2})}$, see Figure 5. Let $a_{1}, a_{2}$ be neighbouring vertices of a regular 15 -gon centered at 0 and having radius $r$. By technical calculations, it turns out that for $r=1.95$ one of the points of intersection of $B\left(a_{1}, 1\right)$ and $B\left(a_{2}, 1\right)$, in the figure denoted by $P_{1}$, lies in $B(0,1)$ and the other one, denoted by $P_{2}$, lies out of $\overline{B(0,2 \sqrt{2})}$. It follows that 15 unit balls cover the boundary of $\overline{B(0,2 \sqrt{2})}$; one more unit ball is needed to cover the middle.

\section{Meyer number for regular polygons}

It is interesting to determine the value of the function $f$ on the simplest two-dimensional shapes, namely regular polygons. As a by-product we derive that the Meyer number for every set $\Omega$ which is not 'far' from a ball is bounded by 8 .

Lemma 7.1. Let $r>c:=2\left(1+2 \cos \frac{2 \pi}{7}\right)^{-1}$. Then there exist points $a_{1}, a_{2}, \ldots, a_{8}$ satisfying

$$
\overline{B(0,2)} \subset B\left(a_{1}, r\right) \cup \cdots \cup B\left(a_{8}, r\right) .
$$

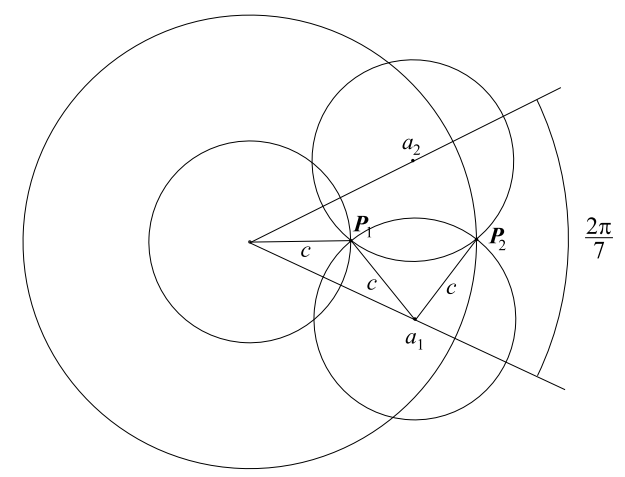

Figure 6. Covering of $\overline{B(0,2)}$ with 8 translated balls $B(0, c)$, see Lemma 7.1. 
Proof. The covering is illustrated in Figure 6. Obviously, it suffices to show that by eight closed copies of the ball $B(0, c)$ one covers $\overline{B(0,2)}$. Put $a_{8}=0$. The centers $a_{1}, \ldots, a_{7}$ of the other copies of the ball $B(0, c)$ are situated on the vertices of a regular heptagon of radius $2 c \cos \frac{\pi}{7}$. Algebraic calculations show that the boundaries of the balls $B\left(a_{1}, c\right), B\left(a_{2}, c\right), B(0, c)$ meet at one point $P_{1}$, and the boundaries of the balls $B\left(a_{1}, c\right)$, $B\left(a_{2}, c\right), B(0,2)$ meet at a point $P_{2}$. Thus $\overline{B(0,2)} \subset \overline{B\left(a_{1}, c\right)} \cup \cdots \cup \overline{B\left(a_{8}, c\right)}$ and the statement of the lemma follows.

Proposition 7.2. Let $\Omega$ be a convex compact set in $\mathbb{R}^{2}$ such that there exist $x, y \in \mathbb{R}^{2}$ and $r>0$ satisfying $\overline{B(x, c r)} \subset \Omega^{\circ} \subset \overline{B(y, r)}$, where $c:=2\left(1+2 \cos \left(\frac{2 \pi}{7}\right)\right)^{-1}$. Then $f(\Omega) \leq 8$.

The above proposition can be easily applied to regular $n$-gons for $n \geq 7$. It remains to determine $f$ for the regular hexagon, pentagon and the square. We do it in a constructive way.

Remark 7.3. For $\Omega$ a regular hexagon, pentagon or square, we have $f(\Omega)=9$, as illustrated in Figures 7, 8, and 9.

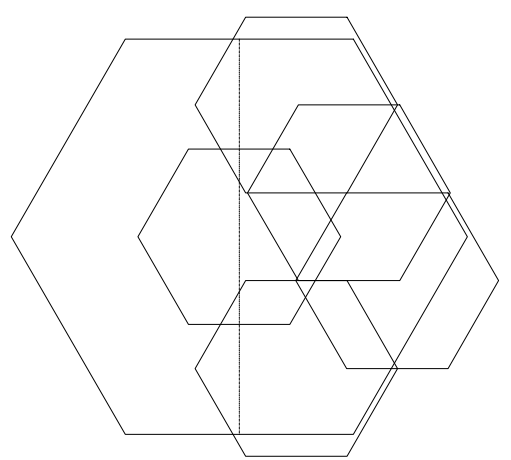

Figure 7. If $\Omega$ is a hexagon inscribed into a circle of radius 1 , then $\Omega-\Omega$ is a hexagon inscribed into a circle of double radius. To cover the boundary of the closed hexagon of radius 2 , we need 8 open hexagons of radius 1 , and to cover the center one more open hexagon of radius 1 is necessary.

We summarize the results about the Meyer number of regular $n$-gons in the following proposition.

Proposition 7.4. Let $\Omega$ be a regular $n$-gon. Then $f(\Omega) \leq 8$ for $n \geq 7, f(\Omega)=9$ for $n=4,5,6$ and $f(\Omega) \leq 13$ for $n=3$.

\section{Conclusions}

In this paper we study the Meyer property of cut-and-project sets. Their structural complexity is dependent on the cardinality of the finite set $F$ satisfying $\Sigma(\Omega)-\Sigma(\Omega) \subset$ $\Sigma(\Omega)+F$. We have transformed the problem to studying the Meyer number $f(\Omega)$ of convex compact sets $\Omega \subset \mathbb{R}^{d}$ defined as the minimal number of open copies $\Omega^{\circ}$ needed 


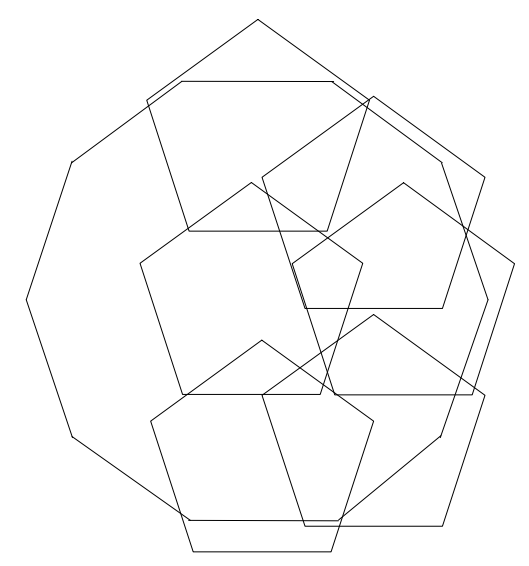

Figure 8. If $\Omega$ is a pentagon inscribed into a circle of radius 1 , then $\Omega-\Omega$ is a regular decagon inscribed into a circle of radius $1+\frac{\sqrt{3}}{2}$. The same explanation is valid as for hexagon.

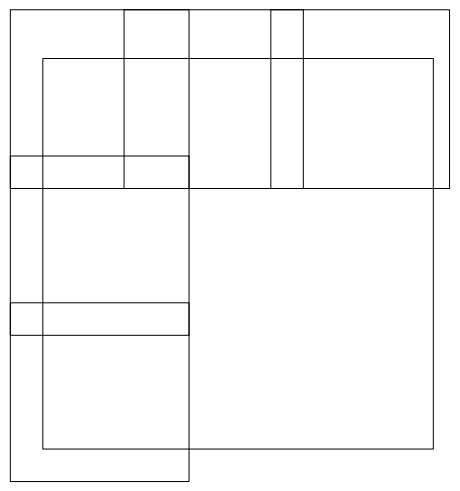

Figure 9. If $\Omega$ is a square having sides of the length 1 , then $\Omega-\Omega$ is a square of double size. To cover the upper side of the closed square of the length 2 we need 3 open squares of half-size. The same for the lower side and the middle side.

to cover the difference set $\Omega-\Omega$. The main result is that for every dimension $d$ there is an upper bound $K_{d}$ such that $f(\Omega) \leq K_{d}$ for any convex compact set $\Omega \subset \mathbb{R}^{d}$. For estimates of $K_{d}$ one needs (according to Corollary 3.3) to find the minimal covering of the closed ball $\overline{B(0,2 d)} \subset \mathbb{R}^{d}$ by unit open balls. This may be a difficult problem in general.

We have focused on dimension $d=2$ and shown that $f(\Omega) \leq K_{2} \leq 26$ for any convex compact set $\Omega \subset \mathbb{R}^{2}$. We can refine the result, if we limit our considerations to centrally symmetric convex compact $\Omega \subset \mathbb{R}^{2}$, for which we have $f(\Omega) \leq 16$. It is however apparent that these bounds are not reached. In order to find better estimates, we have determined the value of the function $f$ for some special types of convex sets in $\mathbb{R}^{2}$. These results lead us to conjecture that $8 \leq f(\Omega) \leq 13$ for any convex compact $\Omega \subset \mathbb{R}^{2}$. For centrally symmetric convex compact sets we conjecture $f(\Omega) \in\{8,9\}$. It would be interesting to study topological properties of the space of those convex centrally 
symmetric sets $\Omega$, for which $f(\Omega)=8$, resp. $f(\Omega)=9$, in particular to describe those $\Omega$ which in the Hausdorff topology form the boundary of classes with different Meyer numbers.

The function $f$ determines the minimal number of open copies $\Omega^{\circ}$ needed to cover the difference set $\Omega-\Omega$. Similarly, one can define a function $g$ as the minimal number of closed copies $\Omega$ needed for covering $\Omega-\Omega$. Obviously $g(\Omega) \leq f(\Omega)$. It is conceivable that the sets $\Omega$, for which $g(\Omega)<f(\Omega)$ are exactly on the boundary between classes of sets with different values of $f$. Upper semi-continuity of the function $f$ could be a useful tool in such a study.

\section{References}

[1] G. Beer, Topologies on closed and closed convex sets, Mathematics and its Applications 268, Kluwer, Dordrecht, 1993.

[2] A. Hof, On Diffraction by Aperiodic Structures, Comm. Meth. Phys. 169, (1995) 25-43.

[3] P. Kramer and R. Neri, On Periodic and Non-periodic Space Fillings of $\mathbb{E}^{m}$ Obtained by Projection, Acta Cryst. A 40, (1984) 580-587.

[4] J. Lagarias, Geometric Models for Quasicrystals I. Delone Sets of Finite Type, Discrete Comput. Geom. 21, (1999) 161-191.

[5] C.G. Lekkerkerker, Geometry of numbers, John Wiley \& Sons, New York 1969.

[6] Y. Meyer, Quasicrystals, Diophantine approximations and algebraic numbers, Proc. Les Houches, March 1994, Beyond Quasicrystals, Les Editions de Physique, eds. F. Axel and D. Gratias, Springer, 1995, pp. 3-16.

[7] R.V. Moody, Meyer sets and their duals, in Mathematics of Long Range Aperiodic Order, Proc. NATO ASI, Waterloo, 1996, ed. R. V. Moody, Kluwer (1996) 403-441.

[8] J. Patera, Noncrystallographic root systems and quasicrystals, in Mathematics of Long Range Aperiodic Order, Proc. NATO ASI, Waterloo, 1996, ed. R. V. Moody, Kluwer (1996) 443-465.

[9] D. Shechtman, I. Blech, D. Gratias, J.W. Cahn, Metallic phase with long range orientational order and no translational symmetry, Phys. Rev. Lett. 53 (1984) 1951-1953.

[10] M. Senechal, Quasicrystals and Geometry, Cambridge Univ. Press, Cambridge, UK, 1995. 